\title{
Technology Roadmapping for Competitive Technical Intelligence
}

\author{
Yi Zhang ${ }^{1,2}$, Douglas Robinson ${ }^{3}$, Alan L. Porter ${ }^{4}$, Donghua Zhu' ${ }^{1}$, Guangquan Zhang ${ }^{2}$, Jie $\mathbf{L u}^{2}$ \\ ${ }^{1}$ School of Management and Economics, Beijing Institute of Technology, Beijing, P. R. China \\ ${ }^{2}$ Decision Systems \& e-Service Intelligence research Lab, Centre for Quantum Computation \& \\ Intelligent Systems, Faculty of Engineering and Information Technology, University of Technology \\ Sydney, Australia \\ ${ }^{3}$ Laboratoire Interdisciplinaire Sciences Innovations Sociétés (LISIS), ESIEE-IFRIS, Université de \\ Paris-Est, France \\ ${ }^{4}$ Technology Policy and Assessment Centre, Georgia Institute of Technology, Atlanta, USA \\ Email Address: yizhang.bit@gmail.com; douglas.robinson@teqnode.com; alan.porter@isye.gatech.edu; \\ zhudh111@bit.edu.cn; Guangquan.Zhang@uts.edu.au; Jie.Lu@uts.edu.au;
}

\begin{abstract}
Understanding the evolution and emergence of technology domains remains a challenge, particularly so for potentially breakthrough technologies. Though it is well recognized that emergence of new fields is complex and uncertain, to make decisions amidst such uncertainty, one needs to mobilise various sources of intelligence to identify known-knowns and known-unknowns to be able to choose appropriate strategies and policies. This competitive technical intelligence cannot rely on simple trend analyses because breakthrough technologies have little past to inform such trends, and positing the directions of evolution is challenging. Neither do qualitative tools, embracing the complexities, provide all the solutions, since transparent and repeatable techniques need to be employed to create best practices and evaluate the intelligence that comes from such exercises. In this paper, we present a hybrid roadmapping technique that draws on a number of approaches and integrates them into a multi-level approach (individual activities, industry evolutions and broader global changes) that can be applied to breakthrough technologies. We describe this approach in deeper detail through a case study on dye-sensitized solar cells. Our contribution to this special issue is to showcase the technique as part of a family of approaches that are emerging around the world to inform strategy and policy.
\end{abstract}

Keywords: Technology Roadmapping; Competitive Technical Intelligence; Text Mining; Tech Mining.

(C) 2015. This manuscript version is made available under the Elsevier user license

http://www.elsevier.com/open-access/userlicense/1.0/ 


\section{Introduction}

Technology Roadmapping (TR) is a future-oriented strategic planning device (Winebrake 2004) that provides a structured approach to help identify relationships between existing and developing technologies, products, and markets, over time (Phaal et al. 2004). If one takes socio-technical change as three interlinked, but distinct, layers (Rip and Kemp 1998), it is reasonable to classify TR endeavours by scope related to these three layers: 1) TR for national Research \& Development (R\&D) planning to inform policy involving economic, scientific, technological, and innovation landscapes; 2) TR for industries and sectors, which focus on existing and potential collaborations and collective coordination in target technological areas; and 3) TR for specific technological trajectories (Zhang et al. 2013).

Due to the strategic emphases, expert knowledge plays a determinant role in TR and the development of TR remains a largely qualitative task (Geum et al. 2015). Traditional text mining techniques, although widely applied for technical characterization, mainly defer to expert contributions in devising TRs (Kostoff et al. 2004). Phaal et al. (2004) summarized fourteen examples of general TR cases to offer a guidebook for TR alternatives. There are also quite a few TRs that rely on quantitative methods with diverse emphases (Gerdsri and Kocaoglu 2007; Lee et al. 2009a; Huang et al. 2014; Zhou et al. 2014; Geum et al. 2015). However, there are still no adaptive criteria and metrics for the selection and evaluation of TRs while applying for actual implementation; existing ones tend to be limited within particular systems. This paper thus focuses on the following research questions:

1) How to balance qualitative and quantitative methodologies to inform TR regarding key components and their relationships?

2) Which criteria and metrics can be used for the selection and evaluation of TR composing models at the implementation stage?

3) How is TR related, similar to, and different from technology foresight projects?

In this paper, we address concerns of Competitive Technical Intelligence (CTI) (Porter and Cunningham 2005) and aim to develop a series of TR models that balance qualitative and quantitative methods. First, based on traditional text mining techniques and a "Term Clumping" stepwise process (Zhang et al. 2014a), we present a term/topic-based TR composing model (Zhang et al. 2013) that highlights the interaction between core technological components. Then, we introduce Subject - Action - Object (SAO) analysis and the Contradiction Matrix concept of TRIZ theory to retrieve Problem \& Solution (P\&S) patterns (Zhang et al. 2014b). Those can contribute to a problem-solving sequence for technological evolutionary pathways in P\&S pattern-based TR model. In parallel, we apply Fuzzy Set theory (Zadeh 1965) to transfer rough expert knowledge to defined numeric values. This can help generate TR automatically (Zhang et al. 2015b).

This paper draws on Science, Technology \& Innovation (ST\&I) data -- e.g. publications, patents, and academic proposals -- to generate historical TRs. We identify developmental patterns and their relationships via text mining and bibliometric techniques, the summarization of which would be used to understand technology evolutionary pathways and to inform R\&D program management. Specifically, our model is to seek approaches, e.g. expert knowledge, trend extrapolation, and quantitative methods, to get from the historical data-based TRs to forecast future developmental trajectories. We then compare the strength and weakness among the above TR models, and propose criteria for selecting the most suitable TR at the implementation stage. It is also beneficial to combine TR models with ST\&I factors, which concentrate on specific research objectives - e.g., Triple Helix model that emphasizes government-industry-academy relationships (Etzkowitz and Leydesdorff 1995; Etzkowitz and Leydesdorff 2000) or the GUISPs model that focuses on the Government-University-Industry Strategic Partnerships (Carayannis et al. 2000); incorporation of multiple ST\&I data types (Zhang et al. 2015b); and attention to the Technology Delivery System (TDS) for market/user, R\&D, and manufacturing factors (Robinson et al. 2013b).

This paper is organized as follows - the Related Work section reviews previous studies on qualitative and quantitative methods for TR. The Methodology section presents three models for composing TRs Term/Topic-based TR, P\&S Pattern-based TR, and Fuzzy Set-based Automatic TR. The Empirical Study follows, applying our TR models to Dye Sensitized Solar Cells (DSSCs) to profile technological evolutionary pathways and foresee possible trends over the near future. We summarize the criteria that could be used for TR selection and evaluation and discuss the similarities and differences between TR and other foresight projects in the Discussion section. Finally, we conclude our research and outline future research priorities. 


\section{Related Work}

This section reviews literatures on Qualitative Methods-based TR, Quantitative Methods-based TR, and Hybrid TR.

\section{Qualitative Methods-based TR}

Since Motorola and Corning first applied TRs for commercial strategy and technology evolution \& positioning studies (Probert and Radnor 2003), TR has become a powerful instrument for supporting strategic planning. This stream keys in exploring the dynamic relationships among technological resources, organizational objectives and the changing environment (Phaal et al. 2004). Qualitative methods -- e.g. expert interview, Delphi, scenario planning, discussion/seminar/workshop -- take leading roles in TR's construction and implementation. These usually involve academic researchers, industrial stakeholders, and government officials (Garcia and Bray 1997; Phaal et al. 2004; Winebrake 2004; Zhang et al. 2013).

As a pioneer of TR studies, Sandia National Laboratories constructed fundamental criteria and schemes for roadmapping (Garcia 1997; Garcia and Bray 1997).Their 3-phase process and its modified versions were applied to a large range of emerging technologies -- e.g. microsystem and nano-system (Walsh 2004), semiconductor silicon industry (Walsh et al. 2005), and pharmaceutical technology (Tierney et al. 2013). Aiming to outline a general guidance to adapt wider strategic needs, Lee and Park (2005) first developed a modularization method-based TR customizing function. Phaal et al. (2006) designed a catalogue for technology management-oriented analytics. Tran and Daim (2008) laid out technology assessment-related approaches for defined levels of public decision making domains and for business and non-government domains. Then, Phaal et al. (2012) proposed a core roadmapping framework for multiple strategic perspectives or a hierarchical family of roadmaps.

What is clear is that, although quantitative methods are increasingly applied to TR, they are outweighed by qualitative methods-based TR which remain the mainstream of current TR activities and, especially, real-world applications -- e.g. manufacturing industry (Gerdsri et al. 2009), internet security technologies (Fenwick et al. 2009), produce-service integration (Geum et al. 2011), car-sharing service (Geum et al. 2014), transparent display (Jeong and Yoon 2015). One reasonable understanding for the popularity of qualitative method-based TR is be that expert knowledge affords powerful credibility to take responsibility for the results; although there is always possible expert biases that could be counterbalanced by quantitative approaches.

Quantitative Methods-based TR

Text mining, as well as bibliometric, scientometric, and informetric techniques have been increasingly used to retrieve textual elements for ST\&I studies since the 1990s (Kostoff et al. 2004). Additionally, computer-based graphical techniques first have been introduced to provide aids for developers and to convey information to users (Walsh 2004). Now, the development of intelligent information techniques -- e.g. artificial intelligence, pattern recognition, and machine learning -- dramatically increases the capability to identify and visualise potential relationships semi-automatically, although this is still far away from standard applications.

Narrowing our focus on ST\&I text analyses, one technique, which is widely recognized, is to retrieve topics via textual elements -- e.g. words, terms, or phrases -- and then to identify their relationships via defined association rules. There has been a substantial contribution in the form of automated techniques, although most of them could only be defined as quantitative methods for information extraction and visualization rather than strictly quantitative method-based TR. As an example, based on co-occurrence analysis, Zhu and Porter (2002) developed a semi-automatic approach to extract and visualise information for network analysis; Chen (2006) developed a general approach to detect emerging trends from co-citation networks and applied this for visualising TR automatically; Waltman et al. (2010) defined an association link to blend linkages -- e.g. co-occurrence, co-citation, and bibliographic coupling -- and visualised grouped nodes as networks. In parallel, novel statistical techniques also started to occupy a position in historical data-based trend analyses. E.g. Allan et al. (1998) proposed approaches to find and follow new events in a stream of broadcast news stories; Kim et al. (2009) complemented a probabilistic approach to retrieve linguistic relationships from patents and discover technological trends; and Blei (2012) applied a Topic Model algorithm to analyze all of the issues of Science magazine from its launch in 1880 to 2002.

Today, the techniques and methodologies for quantitative method-related TR are still under construction. Decades ago Kostoff et al. (2001) asserted that "the proper use of automated techniques 
for text mining is to augment and amplify the capabilities of the expert by providing insights to the database structure and contents, not to replace the experts by a combination of machines and non-experts”; this still pertains for ST\&I studies.

\section{Hybrid TR}

It is commonly accepted that, in a hybrid TR model, computer-based techniques help process massive raw data and reduce scalable data dimensions for further manual operations, and expert-based qualitative methods play active roles in result selection and evaluation.

Kostoff and Schaller (2001), exploring the combination of qualitative and quantitative methodologies, aggregated TR variants into two fundamental TR approaches: expert-based and computer-based, and then, proposed a disruptive TR developing process which introduced the text mining component of Literature-Related Discovery (LRD) to identify technical disciplines and experts and assisted these experts in workshops (Kostoff et al. 2004). Further, the LRD method (Kostoff et al. 2008) has become an effective instrument to link two or more literature concepts that have heretofore not been linked, and is used to assist medical experts to explore potential treatments of quite a few diseases (Kostoff 2014; Kostoff and Patel 2015)., LRD does not link directly with TR development at this moment but it would be reasonable to imagine potential relationships.

Our Forecasting Innovation Pathways (FIP) continues the hybrid TR tradition (Robinson et al. 2013b). Tech Mining (Porter and Cunningham 2004) and science overlay mapping (Rafols et al. 2010) techniques were used to capture R\&D factors and potential commercial signals, and, at the same time, we set expert engagement as a particular step to run through the whole process, feedback in multiple studying iterations, and help create the FIP mapping (Guo et al. 2012; Robinson et al. 2013a). Furthermore, Huang et al. (2012) enriched the expert knowledge into a detailed workshop setting, and Porter et al. (2013) on the one hand, introduced a semantic map to represent patent applications, and one the other hand, illustrated the attempt that combined quantitative results - identified factors - and expert knowledge to refine the FIP mapping in the workshop process. We have applied the FIP approach to address various emerging technology concerns, including case analyses of nano-enhanced solar cells (Porter et al. 2010), nano-enabled biosensors (Huang et al. 2012), hybrid \& electric vehicles (Porter et al. 2013), and nano-enabled drug delivery (Robinson et al. 2013a).

Additional hybrid TR approaches are notable. Yoon and Park (2005) used keywords to help expert-based morphology analysis, then, Lee et al. (2009a) introduced citation-based patent analytic approaches and Pajek to identify relationships among the keywords, which they also shared as a keyword-based patent/knowledge map (Yoon and Park 2005; Lee et al. 2009b).Geum et al. (2015) used co-occurrence analysis to extend the relationship calculation to a two-layer mapping. Another interesting study is that Choi and Park (2009) proposed a citation-based algorithm to link isolated patents as development pathways. This solved the relation identification problem to a certain extent, and experts were invited to select key patents and label clusters.

\section{Methodology}

Our methodology, as introduced in "Related Work," suits the framework of hybrid TR. We take up the challenge to seek a suitable balance between qualitative and quantitative methodologies, engage ST\&I factors for CTI concerns, and develop a strategy and target-driven TR composing method. Our approach includes a Term/Topic-based TR Composing Model, a P\&S Pattern-based TR Composing Model, and a Fuzzy Set-based TR Composing Model. The framework of this paper is given in Figure 1 . 


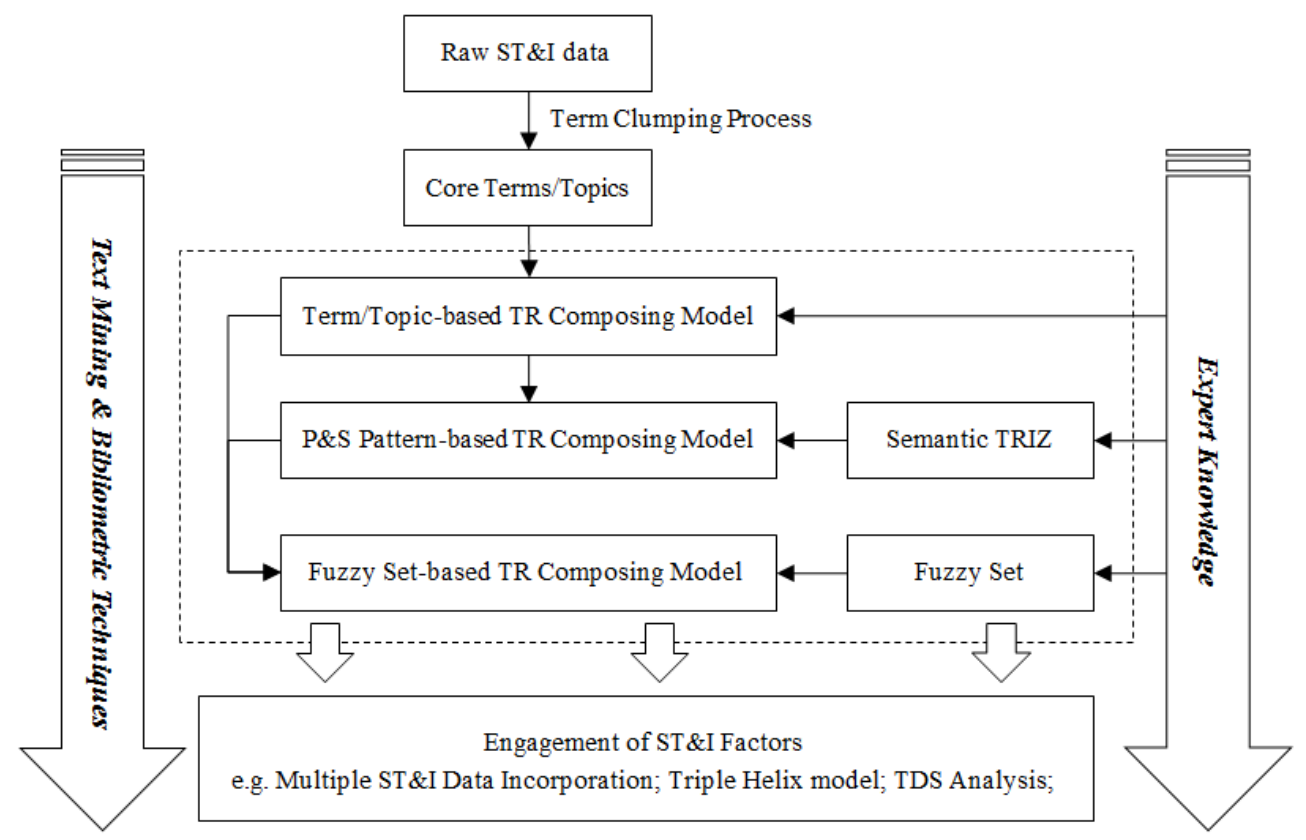

Figure 1 Framework of Technology Roadmapping for Competitive Technical Intelligence

ST\&I textual data is the main input of our method. General ST\&I data includes academic publications, patents, academic program proposals, technical product reports, etc. We mainly focus on the textual content of title and abstract, but other sections, e.g. claims of patent data or full text, also make sense for our scope. We apply a Term Clumping process (Zhang et al. 2014a) to clean, consolidate, and cluster the meaningful terms and phrases retrieved by Natural Language Processing, and the outputs core terms and topics - are considered as the basic elements of our TR models.

\section{Definitions of TR}

Aiding with text mining and bibliometric techniques to explore value-added technological information, our TRs mainly emphasize detailed technological evolution of specified NESTs. We also attend to ST\&I factors on national R\&D and industrial levels. Considering the general format of TR, we define the basic element of TR as the "Object" and denote an Object as $O(L, I, T)$ where $L$ is for Label, $I$ is for Implication, and $T$ is for Time. The Relationship between objects is described as $R\left(O_{i}, O_{j}\right)$. Detailed definitions:

- Object - the element that is laid out on TR and is usually described as the core technological component, e.g. the terms and topics derived from the Term Clumping process, or the P\&S patterns identified via a Semantic TRIZ process;

- Label - the semantic structure of Object that would be a term or a phrase or a sentence;

- Implication - the description of TR's strategy and target foci, e.g. technology development and technology commercialization;

- Time - the time that the Object appeared first in target ST\&I data or first ascended in a "top N" high-frequency terms list and usually would be yearly, semi-yearly, or monthly;

- Relationship - the direct or indirect linkage between Objects, e.g. co-occurrence, semantic similarity, problem and solution, collaboration and competition among target organizations.

We have designed a general format of TR shown as Figure 2, where the horizontal axis and vertical axis denote Time and Implication respectively and compose TR's basic framework. We could divide Implication into several phases to indicate a hierarchical structure for specified strategy or target and we also use a milestone line to highlight a significant event in the past. In particular, we extend the horizontal axis and add a Forecasting section to compare with profiled historical pathways. We also use diverse shapes to distinguish the Object's emphasis for ST\&I factors, e.g. different data sources or different organizations. The linkage among Objects is used to reflect the relationships identified by specified association rules. 


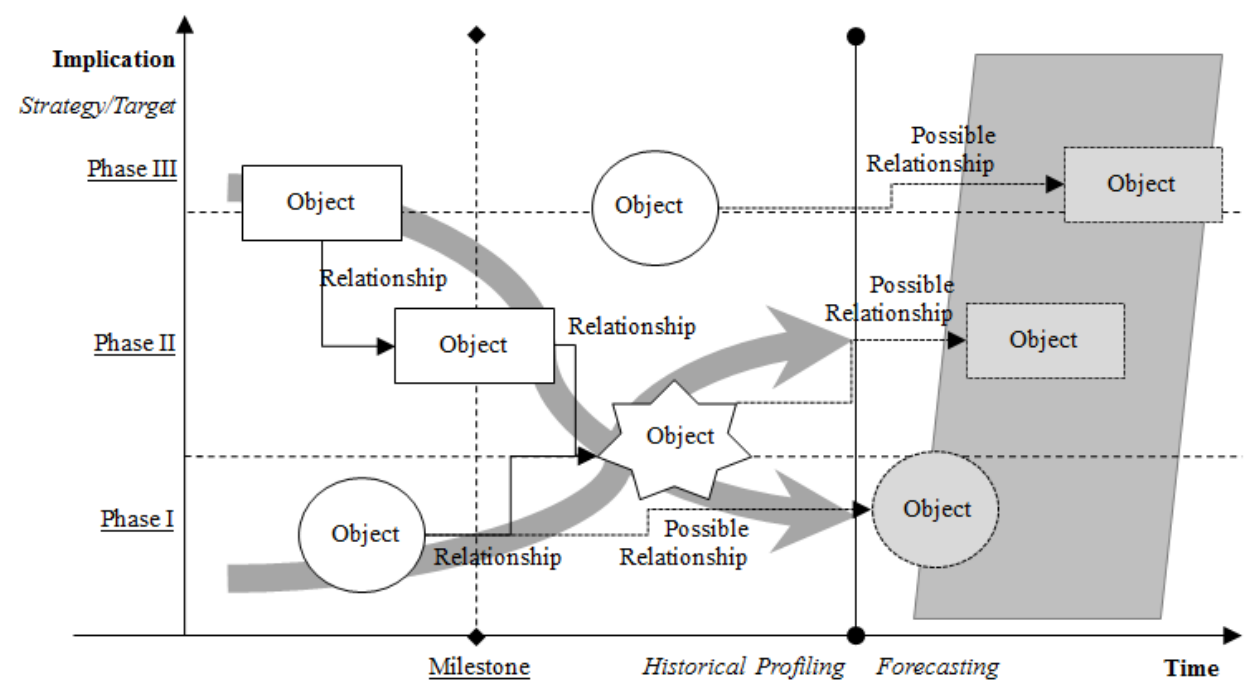

Figure 2 General Format of Technology Roadmapping

Composing Model of TR

Following the definitions and the structure of the general format of TR in Figure 2, we propose three TR composing models with different approaches to identify Object, Relationship, and Implication. First, the term/topic-based model highlights the details of technological components and helps understand a technology evolutionary pathway at a macro level. Then, the P\&S pattern-based model keys on specific technical problems, and attempts to know the dynamic trend on how those problems are solved and the interactions between problems and solutions. The fuzzy set-based model is considered as an aid in a non/few-expert supported environment to increase the adaptability of our TR models.

\section{1) Term/Topic-based TR Composing Model}

The term/topic-based TR composing model is the basic model in our method. We built up a Term Clumping process to clean, consolidate, and cluster terms. Traditional thesaurus, stem-based term consolidation, and association analysis were combined in a stepwise process for identifying core terms from massive ST\&I record sets (Zhang et al. 2014a). We also proposed a data-driven K-Means clustering model attached after such a Term Clumping process to group terms or records as core topics (Zhang et al. 2015a). The above efforts contribute to the pre-processing of this composing model. We then define the core terms and topics derived from Term Clumping process as Objects. We also are able to calculate the relationships among topics via similarity measures, although, sometimes, this kind of relationship is rough and uncertain. We would decide to use the linkage or not. As an option, based on calculated linkages, we locate related Objects on a similar layer and group them together, and then, engage expert knowledge to evaluate the results.

The Objects in this model are defined as core technological components, which mostly relate to the detail of target emerging technologies- e.g. materials, techniques, processing methods, products. This model generally emphasizes a question like: "What the hot technological components were, are, and will be?” which highlights these isolated components and attempts to summarize evolutionary pathways via tracking the changes.

\section{2) P\&S Pattern-based TR Composing Model}

Narrowing our focus into specified technological components, we introduce SAO analysis and the Contradiction Matrix concept of TRIZ theory to enrich the core terms and topics into SAO structures, and identify SAO structures as technical problems or related solutions. This paper mostly follows our proposed methodology for SAO structure retrieval and P\&S pattern identification (Zhang et al. 2014b). The framework of the P\&S pattern-based TR composing model is shown as Figure 3.

In the SAO analysis, we define the core terms and topics as Subject/Object, retrieve their nearby verbs from a combined Title + Abstract field or full text, and define the verbs as "Action," and the entire phrase as an SAO structure. The main concept of the Contradiction Matrix is that external or internal contradictions exist between "object" and "tool," and the way we solve contradictions is the same as how we find "ideal final results” (Rantanen and Domb 2010). We apply this idea to SAO structures and 
introduce expert knowledge to help define them as Problems or Solutions. The P\&S patterns make good sense to identify the relationships between Problem \& Solution, Problem \& Problem (P\&P), Solution \& Solution (S\&S), and Solution \& Problem (S\&P). Zhang et al. (2014b) listed a general definition of the relationship between P\&S patterns: P\&P - Relate, P\&S - Solve, S\&S Relate/Upgrade, and S\&P - Evolve. We would re-define these relationships depending on real requirements.

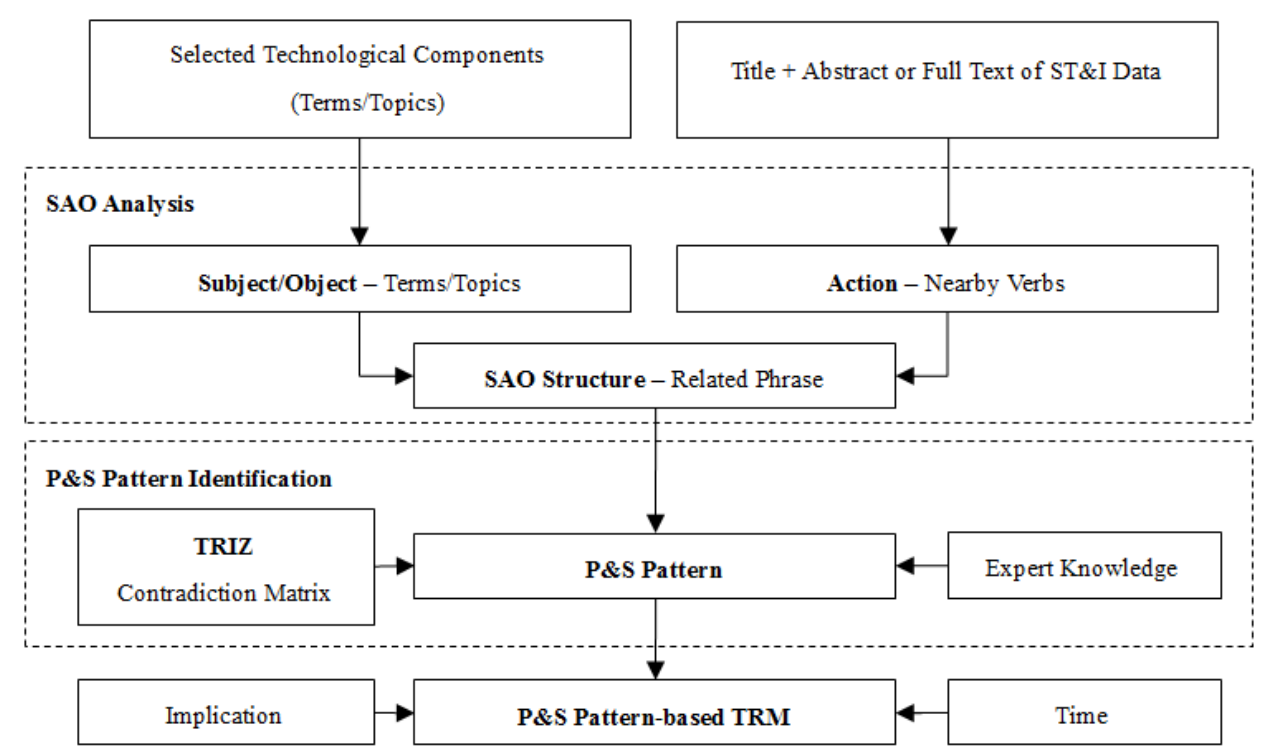

Figure 3 Framework of P\&S Pattern-based TR Composing Model

The P\&S pattern-based TR composing model focuses on the questions: "Which problems are addressed, and when?" and "How the problem was solved (new techniques, materials, or something else), and when?” Comparing with the term/topic-based TR, this model helps discover linguistic features of technological components and emphasizes the logical relationships during the process of technology evolution.

\section{3) Fuzzy Set-based TR Composing Model}

The fuzzy set-based TR composing model could be considered as an assisting instrument for the above two models. It is easy to define the Time as the coordinate figure of the horizontal axis in TR, but the Implication - our strategy and target - usually is vague and difficult to be defined as exact numeric values. Therefore, in the above two models we draw the TRs manually to adapt the vagueness and uncertainty. Aiming to minimize the need for aid of manual operations and maximize the usage of expert knowledge in limited time and scope, this model introduces the fuzzy set to transfer vague human thoughts (e.g. expert knowledge) to defined numeric values and helps generate a TR automatically.

We denote "all Objects" as the universe $X=\left\{x_{1}, x_{2}, \ldots, x_{i}, \ldots, x_{n-1}, x_{n}\right\}$ and "each phase of the Implication" as a fuzzy set $A_{j}$ defined on the $X$ where $j \in[1, m]$, where $m$ is the number of the phases. The membership function $A_{j}\left(x_{i}\right)$ is considered as the degree that Object $x_{i}$ belongs to the phase $A_{j}$, and will be decided depending on research purposes and empirical data.

\section{Engagement of ST\&I Factors}

Current CTI studies involve a wide range of ST\&I factors, which address real-world concerns for diverse targets and emphases -- e.g. who plays dominant roles in specific domains? What are the interactions among selected organizations - collaboration or competition? What benefits would we gain from multiple ST\&I incorporation? And how to visualize the time gap among selected ST\&I data sources? We are able to imagine valuable outputs from the combination of these ST\&I factors and TR models -- e.g. to enrich TR's content for meaningful information, and to explore underlying relations among TR's Objects. This section attempts to engage several ST\&I factors with TR models for further CTI study. 


\section{1) Triple Helix Model/ GUISPs Model}

General TR studies follow the details of a specific technology closely, and ignore the holders of that technology. However, exploring key players and collaborative/competitive relationships in specific technology arenas is one important topic for CTI studies, and it is helpful to introduce this issue to TR model to extend current scope.

The Triple Helix model holds interest as to research knowledge transfer among university, industry, and government sectors (Etzkowitz and Leydesdorff 1995; Etzkowitz and Leydesdorff 2000). It is also a feasible instrument to measure ST\&I activities in many domains (Park et al. 2005). Similarly, the GUISPs model focuses on R\&D strategies and emphasizes knowledge integration through company partnerships with government agencies, universities, and other industry players (Carayannis et al. 2000). Considering the general format of TR in Figure 2, we reserve an interface to describe the information of Triple Helix model/GUISPs models as shaping target Objects.

Generally, organization information is available in discrete fields in ST\&I records -- e.g. giving the affiliation of a publication's author, or patent assignee, and we define the organization as the one who published the records containing our target Object. Therefore, it is easy to retrieve the organizational information via text mining techniques (usually Natural Language Processing (NLP) techniques), and then, we are able to extend the definition of Object $O(L, I, T)$ to $O(L, I, T$, Org.). In this context, our TR models describe the fourth dimensional information of Object via its shape, and indicate the interactions according to the Relationships among Objects with the same or different shapes.

\section{2) Multiple ST\&I Data Types}

There are numerous available ST\&I data sources with varying emphases. Zhang et al. (2015b) summarized popular ST\&I data in current researches and classified their emphases into four levels Idea/Concept, Basic Research, Application Research, and Commercial Information. It is also critical sometimes to combine those emphasis and different types of ST\&I data together. E.g. to explore the time gap between idea and commercialization, looking at both R\&D publication compilations and patent datasets can illuminate commercialization factors along with technical ones (Zhang et al. 2013; Zhang et al. 2015b).

We extend the definition of Object $O(L, I, T)$ to $O(L, I, T, D)$ where $D$ is used to describe data type and match with the shape of Object. We can imagine the benefit of this kind of combination as the multiple ST\&I data-based TR opens a window to compare ST\&I activities of target technologies at different levels. We suggest considering the diverse emphases of different ST\&I data types.

\section{3) Technology Delivery System (TDS)}

TR is developed to trigger change, whether internally in an organization or to create changes to industries and value chains. For the latter, we apply what we call a TDS, which depicts a value chain as a system diagram of components that come together to form a functioning value chain. Below is an example for lab-on-a-chip technology (shown as Figure 4).

In this example, the multi-disciplinary science community interacts in various ways to create the knowledge that forms a knowledge reservoir that serves as a resource for innovation (central/left-hand side of the diagram). This knowledge can be mapped using tech mining tools (Porter and Cunningham 2004). Industrial actors can be identified, and here they are instrument manufacturers and the pharmaceutical industry. These represent the firms which add value to the knowledge from the reservoir, through developing manufacturing techniques and standards. However, the situation is more complex, there are start-ups involved and hospitals as both co-developers and users of the innovations coming from lab-on-a-chip roadmaps, and thus the value chain is more extended. In addition there are other sectorial/application drivers (bottom of the diagram) which create expectations and hype. These may trigger further "fishing" in the knowledge reservoir, or indeed trigger knowledge production there. Another part of the TDS is the framing conditions, funding agencies, insurance companies (if they are relevant for the value chain), organized user groups which drive demand (here it is patient groups), and regulatory agencies.

The TDS can be used both as a way of depicting the elements and relationships in the situation today as well as a way of mobilising the insights from technology roadmapping to help depict "a future working world.” Put another way, the desired future value chain will entail a future TDS. In this way, it is much more than a picture of boxes and arrows, but it is a translation tool of the roadmapping procedure to those not involved in the roadmapping. The TDS helps inform, justify, and evaluate the implementation of TRs. 


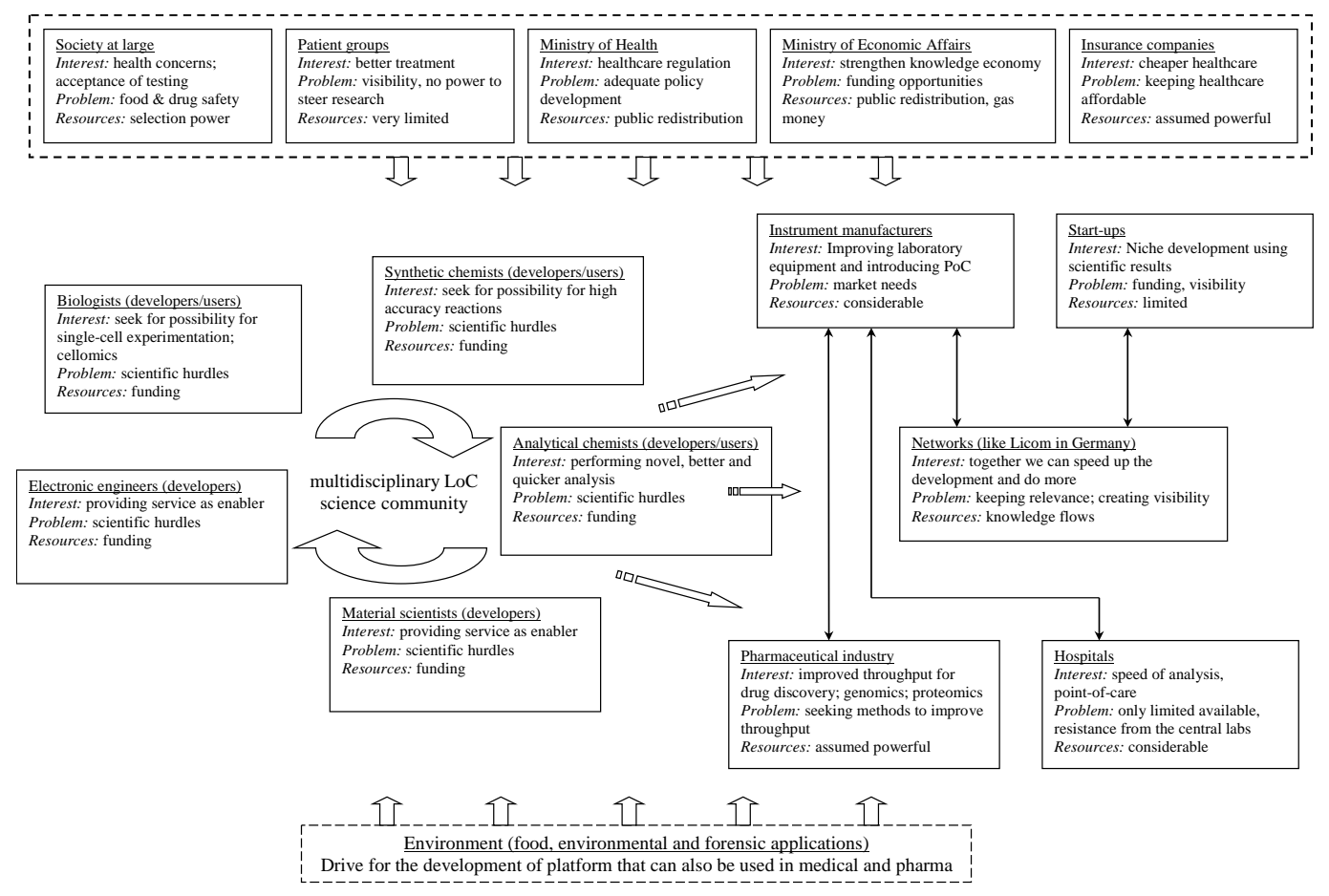

Figure 4 A TDS for the emerging lab-on-a-chip field (Probert and Radnor 2003)

\section{Empirical Study}

In this section, we apply our method to Dye Sensitized Solar Cells (DSSCs). Here, we 1) blend the term/topic-based TR with the fuzzy set-based TR on Web of Science (WoS) publication data, and automatically generate one TR of global DSSC development that emphasizes the detailed technological components of DSSCs, e.g. materials, techniques, components, and products. We then, 2) address the potential collaboration and competition among government, university, and institutions to the P\&S pattern-based TR with Derwent Innovation Index (DII) patent data. The combination of the two approaches adds power to illustrate solving pathways for specified technological problems and also indicates relationships among related organizations.

\section{Global DSSCs TR}

Publication data focuses on basic technological components and acts as the first step to transfer innovative ideas to applications (Zhang et al. 2015b). Thus, aiming to landscape the global DSSC technological development pathways, we select DSSC-related scientific publications in Web of Science (WoS). Since DSSCs were invented in 1991 and are still a strongly emerging Solar Cell technology family, we updated our DSSC dataset and captured 13,066 records from 1991 to 2014. Applying our Term Clumping process (Zhang et al. 2014a) then helped us to retrieve 447 core terms from a raw 191,186-term list -- the stepwise results of which are shown as Table 1.

Table 1 the Stepwise Results of Term Clumping Process for the Global DSSCs TR

\begin{tabular}{|c|l|c|}
\hline No. & \multicolumn{1}{|c|}{ Step } & $\# T^{*}$ \\
\hline 1 & Raw dataset for 13,066 publications & 191,186 \\
\hline 2 & Basic Cleaning - removed common terms, e.g. the, what, he. & 147,946 \\
\hline 3 & $\begin{array}{l}\text { Basic Cleaning - removed meaningless terms in academic and scientific } \\
\text { publications, e.g. method, introduction, approach. }\end{array}$ & 144,820 \\
\hline 4 & Fuzzy Matching - The stem-based term consolidation & 127,651 \\
\hline
\end{tabular}




\begin{tabular}{|c|l|c|}
\hline 5 & Pruning* - removed terms appearing in only one record & 25,800 \\
\hline 6 & $\begin{array}{l}\text { Combine Term Network Analysis - Association rule-based term } \\
\text { consolidation, where low-frequency terms were consolidated to the } \\
\text { high-frequency terms that always appear together }\end{array}$ & $\mathbf{1 8 , 5 1 5}$ \\
\hline 7 & $\begin{array}{l}\text { Term Cluster Analysis - Association rule-based term consolidation, where } \\
\text { the number of sharing words was used to calculate the similarity between } \\
\text { terms, and then we consolidated similar terms to the most representative one }\end{array}$ & 447 \\
\hline 8 & $\begin{array}{l}\text { Topic Cluster - grouped terms into a number of topics via K-Means cluster } \\
\text { algorithm (Zhang et al. 2015a), and selected the topic terms of each cluster }\end{array}$ & 4 \\
\hline
\end{tabular}

\#T: The total number of terms left after this step;

Pruning: The following steps after Step 5 were mainly based on co-occurrence analysis, and the terms appearing in only one record would make no sense there. Thus, we remove all those terms although there would likely be some low-frequency terms containing innovative information.

In this case, we emphasized the technological evolution process of DSSCs, and after consulting with domain experts, a general understanding was that DSSC-related techniques usually started with innovation in materials, and then, novel techniques were used to manufacture middleware components, and new products appeared finally. Therefore, we decided to build up three fuzzy sets for material, technique \& component, and product to indicate the degree of technology development - the phases. At the same time, we evaluated the feasibility of introducing machine learning techniques to train the membership functions of the three fuzzy sets. Machine learning technique matched well with our scope, but the difficulty in training an algorithm to understand complex linguistic and technological relationships would extraordinarily increase the uncertainty of our results. In this context, based on expert knowledge, we let the power set $F(X)=\left\{A_{1}, A_{2}, A_{3}\right\}$, and chose a Gaussian distribution to identify the membership functions as below.

$$
\begin{gathered}
A_{1}(x): X \sim N(0,1 / 2 \pi), x \in[0,1] \\
A_{2}(x): X \sim N(1 / 2,1 / 2 \pi), x \in[0,1] \\
A_{3}(x): X \sim N(1,1 / 2 \pi), x \in[0,1]
\end{gathered}
$$

We first convened experts to classify the 447 terms into one of the three fuzzy sets and marked a membership grade $A_{j}(x)$ for the selected fuzzy set $A_{j}$. One term could indicate both material and technique, or both technique and product, and experts help enforce one category, but fuzzy sets reflect the degree of belonging. As an example, because of diverse background, some experts categorized the term "electron injection" as technique \& component and marked the membership grade as 0.95, while some others marked it as 0.6 to the fuzzy set - material because they thought it related to material more, but not fully. We then used the membership function conversely to calculate the $x$ value which was identified as the degree of technology development. Furthermore, the mean of the $x$ values derived from different experts was set as the Object's Y value on the mapping, while its $\mathrm{X}$ value was the first year when it appeared in the dataset.

The term/topic and fuzzy set-based TR for global DSSCs development is given in Figure 5 (from 2008 to 2014 as an example). It is obvious that this mapping explores the detailed DSSC technologies during its past 24-year development pathway, but professional expertise would be necessary to understand thoroughly. Aided by domain experts, we address several concerns:

1) The preparation material of DSSCs was always a hot topic for researchers. The first peak time was from 1996 to 2000, when nanotechnology was introduced to prepare electrode and electrolyte in 1998, and then, the second peak time came with the invention of graphene in 2004 which first appeared in our list as graphene film four years later. The combination of these two emerging technologies captured the interests of researchers in the following years. TiO2 and $\mathrm{ZnO}$ are still the leading materials for DSSCs, and, since 2010, various kinds of materials have been applied as composite or mix materials.

2) Device durability and low-cost fabrication were two main problems during the first 15 years of DSSC development, but environment-friendly features have become a popular and emergent requirement since the term "environmental purification" first appeared in 2012. In parallel, more and 
more one-step or two-step had being used to name related techniques and methods since 2009, which seems to indicate the needs for rapid and convenient DSSC fabrication.

3) DSSC-related techniques concentrated on solar cells, rather than other industrial applications, but drug delivery and biomedical appeared in 2009 and 2011 respectively which would be a good starting point for extended applications of DSSCs. Although experts explained this phenomenon was due to the policy restrictions in most European countries and Japan, note here one argument for rare applications in our results is that we would likely over-clean the records and terms before 2011.

4) Dye is the highest frequency term in the mapping and there is no doubt that it is the most important sub-technology in DSSCs. Organic dye and metal complex dye were two leading directions through 2014, where researchers attempted to broaden the selection of organic or metal substances. 


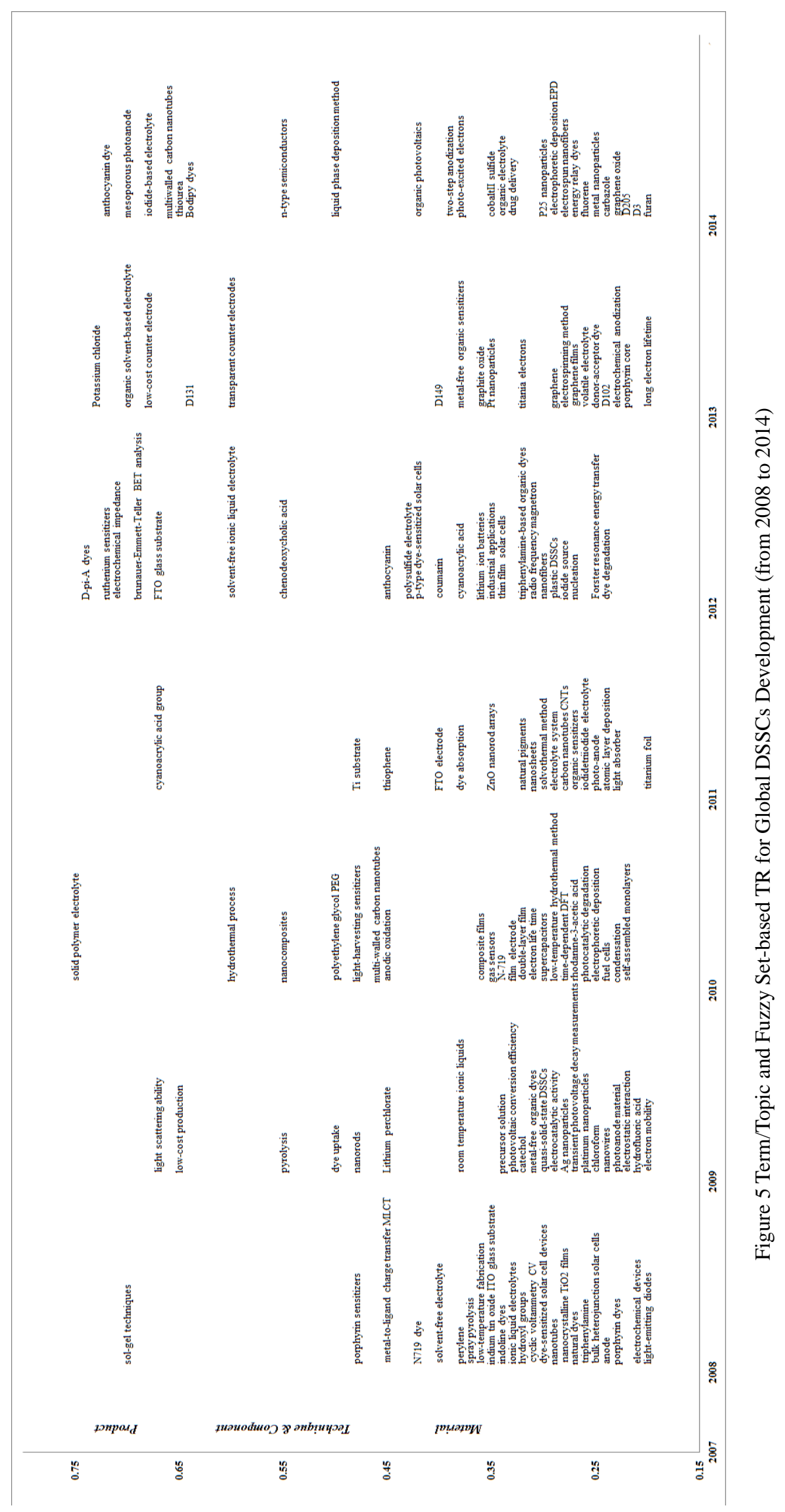


Universities and research institutions lead in research publications, while governments and companies hold more interests in patents due to their commercial concerns. Therefore, we choose the DII patent data for exploring the Triple Helix relationships among the government, university, and company sectors in China's DSSC industry. Our motivation to pursue the Triple Helix Model here is to model the roles that government, academy, and industry play in China's DSSC industry and their possible interactions. Based on our previous experiences, we 1) divided the Academy part of the original Triple Helix model into academy and university to distinguish their distributions; 2) set Chinese Academy Science (CAS) as a special category to highlight this academic institution which has a significant government-support base; and 3) aiming to demonstrate the advantage of the P\&S pattern-based TR on tracking problem-solving pathways, narrowed our focus to the "conversion efficiency" problem, which is considered as a problem throughout the entire development pathway of DSSCs.

With the aid of software, we retrieved 186 SAO structures from the raw content of 1,167 patent records, the priority country of which is China. We followed a similar process in the Global DSSC TR to determine the phases of the Implications - our expert panel discussed the macro-level status of the solutions of "conversion efficiency" problems in China, and then, summarized the phases as Material, Method, and Device. We also arranged the domain experts to evaluate these SAO structures and identify 74 solutions and their relationships. We ignored the Object's shape for a clearer graph and marked the Triple Helix category as the initial letter: University - U, Academy (Institution) - A, Government - G, Industry - I. We present our results in Figure 7. This TR drew a landscape to help understand how the conversion efficiency problem was being solved in China. It represents how the key players of China's DSSC industry focused on certain techniques, and engaged in relationships in the Triple Helix model. Our discoveries are listed below:

1) In China, the "conversion efficiency” problem was solved in four directions - a) to absorb more light via enlarging surface area, adding more layers, etc.; b) to improve the efficiency of the dye, where organic materials and metals were the two foci; c) to improve the efficiency of the film, anode, or electrode, where $\mathrm{TiO} 2$ and $\mathrm{ZnO}$ were two basic materials, nano materials were widely used, and graphene was becoming popular; and d) to transfer the state of the electrolyte from liquid to gel and solid.

2) The "measurement of conversion efficiency" was a focal problem, while we were also able to identify "electrolyte leaking”, "device stability" and "photoelectron transport loss" as relevant problems. Actually, one patent would sometimes focus on another problem and the "conversion efficiency" problem was treated in addition.

3) The University group was the main force of China's DSSC research, which stated 53 solutions in the past 10 years. CAS followed with 10 solutions, while the Industry group had 9 and the Academy had 2. From Figure 6 and experts' experiences during the evaluation process, we take the attitude that the patents of China's Universities generate more innovative techniques than other organizations, and Industry paid more attention to the preparation methods.

4) China's government is considered as the most powerful driving force for China's DSSC development. On the one hand, CAS, the leading organization with the largest numbers of solutions in the map, definitely contributed excellent work for solving the "conversion efficiency" problems. Its government background and the massive program and funding support obtained from government make us believe that China's government was highly involved in the DSSC industry. On the other hand, comparing our analytic results for the data before 2012 (Zhang et al. 2014c), in 2014 the Government finally directly appeared in China's DSSC patents for the conversion efficiency problem. We traced this patent and noticed that it is by the National Center for Nano Science and Technology, China, an academic research centre built by CAS and the Ministry of Education, (that is the reason we only marked it as A/G on the mapping). Nevertheless, we foresee that more state-owned enterprises or institutions are apt to support China’s DSSC development.

5) Collaborations among the Triple Helix organizations in China are rare. Only two solutions were from collaborations between a University and Industry - University of Fudan with Changzhou Youze Technology Co. Ltd., and Dalian University of Technology with Yingkou Opvtech Energy Co. Ltd. It is obvious that geo-advantage played an important role in both collaborations. 


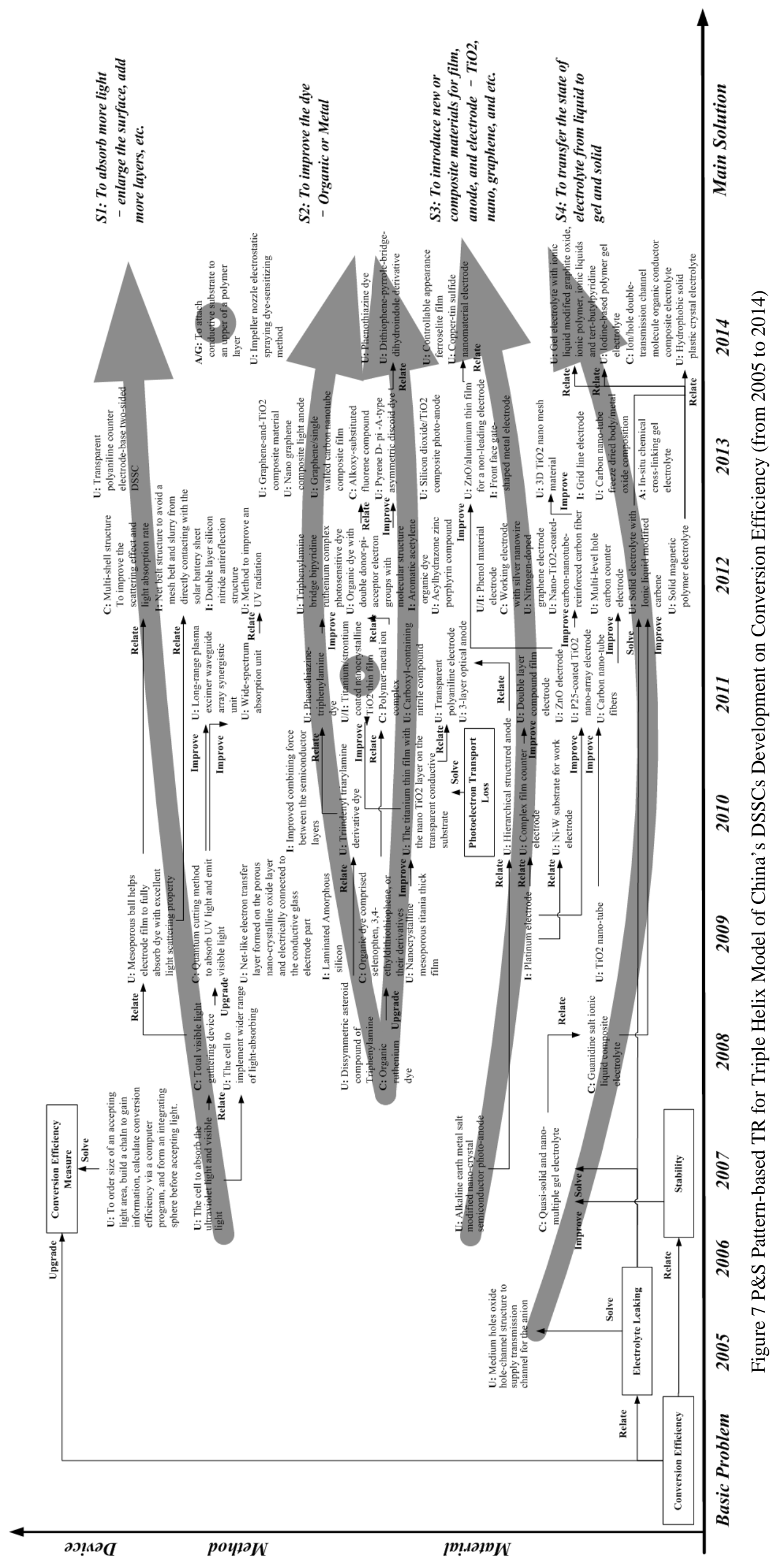




\section{Discussion}

Referring to our TR composing models and the results obtained in the empirical study, we summarize the criteria for TR model selection and evaluation and discuss the similarity and difference between TR and other foresight projects in this section.

\section{Criteria and Metrics}

How to choose the best TR model for specified requirements and evaluate the efficacy of TR are main research questions in this paper. Actually one possible answer would relate to the balance between qualitative and quantitative methodologies. Researchers always argue the subjective bias of expert knowledge and the uncertainty of data evidence, but both heavily influence the implementation of TR, including selection and evaluation.

\section{1) Criteria for Expert Engagement}

We engage experts in various kinds of ways -- e.g. questionnaire, interview, seminar, and workshop. There are two criteria that we need to take into consideration - background relevance and cost (including time and expense). The ideal situation is the background of an expert is highly relevant and the cost is low, however, experts usually are busy, expensive, and might not match one's need exactly. At this stage, in Table 2 we summarize the criteria for expert engagement in possible TR building situations.

Table 2 Criterion for Expert Engagement

\begin{tabular}{|c|l|l|}
\hline Level & \multicolumn{1}{|c|}{ Criterion } & \multicolumn{1}{c|}{ Situation } \\
\hline A & $\begin{array}{l}\text { Ideal Situation - to engage expert knowledge as } \\
\text { much as you can }\end{array}$ & $\bullet$ High Relevance and Low Cost \\
\hline B & $\begin{array}{l}\text { Good Situation - to emphasize expert } \\
\text { knowledge aiding, with limited quantitative } \\
\text { methods }\end{array}$ & $\begin{array}{l}\text { ・ High Relevance and Normal Cost; } \\
\text { - Normal Relevance and Low Cost; } \\
\text { Cost }\end{array}$ \\
\hline C & $\begin{array}{l}\text { Normal Situation - to restrict use of expert } \\
\text { knowledge and prefer quantitative methods }\end{array}$ & $\begin{array}{l}\text { ・ High Relevance and High Cost; } \\
\text { Normal Relevance and High Cost }\end{array}$ \\
\hline D & $\begin{array}{l}\text { Bad Situation - to apply quantitative methods as } \\
\text { much as you can (highly intelligent IT } \\
\text { techniques might be necessary) }\end{array}$ & $\bullet$ Low Relevance \\
\hline
\end{tabular}

The promise here is that the more experts we have the more creditability we are able to gain, and we prefer expert-based qualitative methodologies rather than machine-based quantitative methodologies. As shown in Table 2, we classify the possible real situations into four types with respect to access to expertise - Ideal, Good, Normal, and Bad. In the Ideal and Good situations, we hold the capability to arrange large-scale expert engagement for our studies, and quantitative methodologies would only act as an adjutant for expert-based analyses. Mostly these situations occur in the R\&D planning of national governments or big business, and they would be time-consuming. The situation common in institutions and small \& medium enterprises finds the resources are limited and it is necessary to substitute for experts via quantitative methodologies. However, a systematic research framework construction and feasibility study are necessary, and appropriate expert engagement would be a good guarantor for outputs. We do not suggest persisting in continuing the research in the Bad situation, unless it is possible to introduce highly intelligent IT techniques to fully support the study.

Referring to Table 2, it is helpful to think about expert resources and budget before we start a TR study, and to know what balance between qualitative and quantitative methodologies is fit for our current situation. That would be the pre-assessment for TR selection and evaluation, since we need to know what is on hand.

2) Criteria for TR Selection and Evaluation

This section compares our three TR models with qualitative method-based TRs, quantitative method-based TRs, and hybrid TRs in Emphasis, Situation Requirement, Data Quality, Flexibility (e.g. combining with ST\&I factors, implementing for real-world applications), Visualization, and Understanding (as shown in Table 3). As discussed in "Related Work," the qualitative method-based 
TRs are mainly based on expert knowledge and might involve limited statistical models for computation; the quantitative method-based TRs are mostly automatic generated mappings aiding with text mining, bibliometrics, and other intelligent techniques, and there is little expert engagement for them. The hybrid TR combines them both to a certain extent. We lay out factors that influence the efficacy of TR, and propose criteria for TR selection and evaluation below:

The qualitative method-based TRs hold high flexibility while designing and implementing, and even do not need data support. Their visualization efficacy depends on needs, which sometimes are graph-based but sometimes are text-based. The visual approach would also influence the difficulty of understanding, although most of them are easy-going for governors, administrators, and ones with limited expertise. However, the guarantee from expert participation ensures the credibility of outputs, and the qualitative method-based TRs are still the most popular formats in current implementation.

The quantitative method-based TRs highly depend on relevant algorithms and data. They can be highly effective for data analyses and visualization. However, the algorithms sometimes strictly limit the TRs in design framework and would not be flexible for different data types, graphic formats, etc. Understanding this kind of TRs would need professional expertise. Nevertheless, possible missing relationships among items on the mapping could lead to misunderstanding. It is also critical to believe in computer-based, real-world applications.

The hybrid TRs would be a middle way between qualitative method-based TRs and quantitative method-based TRs and obtain benefits from the both. One challenge of hybrid TRs that we need to consider is how to adapt the different requirements and backgrounds of a number of disciplines, e.g. computer science, technology management, and the target technology.

The fuzzy set-based TR, similar with quantitative-based TRs, fixes elements into a designed basic graph, prefers high data quality, and is not quite flexible for changing requirements. Its advantage would be the possible adaptability for the Bad situation of Table 1 -- e.g. only have very little expert access or expert backgrounds are less relevant to the target. At this stage, it would likely be promising that fuzzy set collects and transfer the rough and limited knowledge as a group decision;

The term/topic-based TR offers most benefits of hybrid TRs, where the appropriate expert engagement reduces the requirements on data quality and increases the flexibility for real-world applications. However, this model mainly focuses on technological components, and the relationships among technological components are derived from quantitative analyses, which sometimes would be rough and uncertain. The P\&S pattern-based TR increases expert engagement to help explore relationships among technological components and its focus on problem-solving pathways also increases the visualization efficacy.

Generally, we need to pay regard to current resources and foci of TR studies, and Table 3 would act as a guide that provides feasible criteria for TR selection and evaluation. 


\begin{tabular}{|c|c|c|c|c|c|c|}
\hline 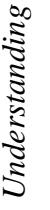 &  & $\begin{array}{l}\text { 莺 } \\
\text { 惫 }\end{array}$ & & 㟒 &  &  \\
\hline 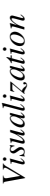 & 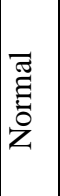 & $\begin{array}{l}\square \\
\\
0\end{array}$ & $\begin{array}{l}\overline{\widetilde{Z}} \\
\tilde{E} \\
0 \\
z\end{array}$ & 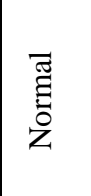 & $\begin{array}{l}8 \\
8 \\
0\end{array}$ & $\begin{array}{l}\overline{\widetilde{Z}} \\
\text { हे } \\
\text { Z }\end{array}$ \\
\hline 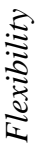 & 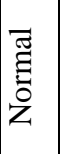 &  & $\begin{array}{l}3 \\
0 \\
0 \\
-1\end{array}$ &  & 芆 & 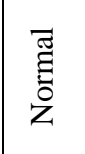 \\
\hline 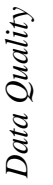 & $\begin{array}{l}\overline{\widetilde{\widetilde{G}}} \\
\overline{\tilde{z}} \\
\mathrm{z}\end{array}$ & 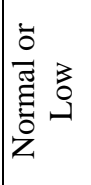 & \begin{tabular}{|l}
$\overrightarrow{\widetilde{T}}$ \\
$\vec{E}$ \\
$\overline{0}$ \\
$z$
\end{tabular} & $\underset{\mathrm{z}}{\mathbb{z}}$ & 모 & 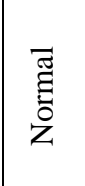 \\
\hline 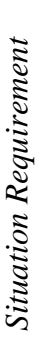 & 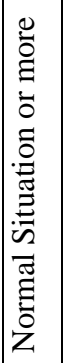 & 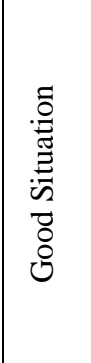 & 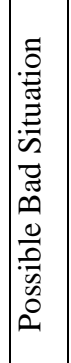 & 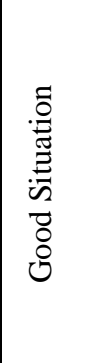 &  & 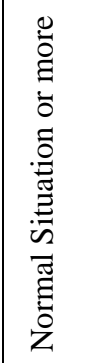 \\
\hline 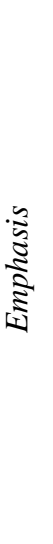 &  &  & 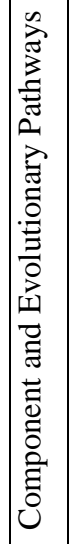 & 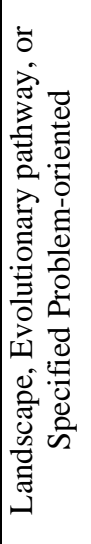 & 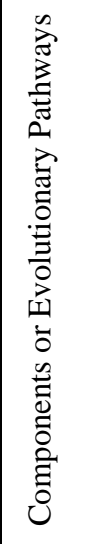 & 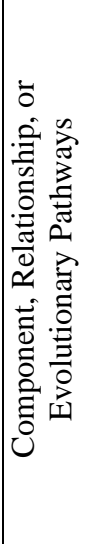 \\
\hline  & 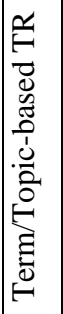 & 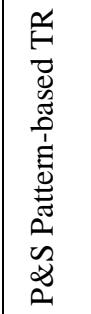 &  & 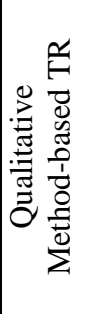 & 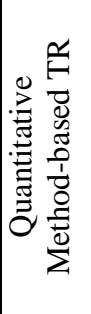 & 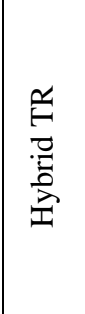 \\
\hline$\dot{z}$ & - & $N$ & $m$ & $\nabla$ & เ & 0 \\
\hline
\end{tabular}




\section{Comparison between TR and Other Foresight Projects}

As shown in the general format of TR in Figure 2, we attempt to combine historical data profiling and forecasting studies within a TR model, which seems to be evidence to indicate a high relevance between TR and foresight studies. Based on Table 3, we list the similarity and difference between TR and other foresight projects in Table 4.

Table 4 Comparison between TR and Other Foresight Projects

\begin{tabular}{|c|c|c|c|c|}
\hline No. & Factors & $T R$ & Expert-based Foresight & $\begin{array}{l}\text { Quantitative-based } \\
\text { Foresight* }\end{array}$ \\
\hline 1 & Future-oriented & One of main foci & Main focus & Main focus \\
\hline 2 & Visualization & Mapping-preferred & No special pursuance & $\begin{array}{l}\text { No special pursuance, } \\
\text { but some prefer }\end{array}$ \\
\hline 3 & $\begin{array}{l}\text { Historical Data } \\
\text { Profiling }\end{array}$ & $\begin{array}{l}\text { One of main foci, } \\
\text { profiling historical } \\
\text { data for assessment } \\
\text { and forecasting }\end{array}$ & $\begin{array}{l}\text { No special pursuance, } \\
\text { but emphasizing the } \\
\text { logical relation } \\
\text { between the past and } \\
\text { future }\end{array}$ & $\begin{array}{l}\text { Recognizing and } \\
\text { learning from } \\
\text { historical data for } \\
\text { prediction }\end{array}$ \\
\hline 4 & $\begin{array}{l}\text { ST\&I } \\
\text { Factor-involved }\end{array}$ & $\begin{array}{l}\text { Concentrating on } \\
\text { specified one or } \\
\text { two ST\&I factors }\end{array}$ & $\begin{array}{l}\text { Involving with ST\&I } \\
\text { factors as more as } \\
\text { possible }\end{array}$ & $\begin{array}{l}\text { Ignoring possible } \\
\text { influence from ST\&I } \\
\text { factors }\end{array}$ \\
\hline 5 & $\begin{array}{l}\text { Expert } \\
\text { Engagement }\end{array}$ & $\begin{array}{l}\text { To seek suitable } \\
\text { balance between } \\
\text { quantitative and } \\
\text { qualitative } \\
\text { methodologies }\end{array}$ & $\begin{array}{l}\text { Emphasize } \\
\text { quantitative-based } \\
\text { prediction models*, or } \\
\text { highly expert-engaged }\end{array}$ & $\begin{array}{l}\text { Almost no } \\
\text { expert-required }\end{array}$ \\
\hline
\end{tabular}

*The quantitative-based foresight mainly indicates mainstream algorithms and models in artificial intelligence, data mining, and machine learning domains -- e.g. link prediction (Liben-Nowell and Kleinberg 2007), burst detection (Bogard and Tiederman 1986), collaborative filtering (Resnick et al. 1994), recommender systems (Resnick and Varian 1997), and time series analysis (Hamilton 1994).

Actual, current foresight projects are divided into two isolated types: expert-based foresight and quantitative-based foresight. R\&D managers prefer qualitative methods for forecasting, which ensures the credibility and meets the needs from a macro level -- e.g. national strategy. In contrast, researchers in mathematics and computer science endeavour in machine-based methodologies to predict events or trends in the near future -- e.g. energy demand and economy time series. These models are limited in solving real-world problems.

It is obvious that TR shares many foci with expert-based foresight projects -- e.g. future-oriented, ST\&I factor-involved, and expert-preferred, but the "roadmapping" feature of TR emphasizes the visualization process and historical data profiling. At the same time, since TR models seek to introduce quantitative methodologies to support decision making, one feasible application is to use statistical models of quantitative-based foresight to help experts foresee possible changes. At this stage, TR seems to be a bridge that connects isolated expert-based foresight and quantitative-based foresight projects.

\section{Conclusions}

In this paper, we have presented an approach to TR that builds on a family of techniques both quantitative and qualitative, created a typology of tools, and applied them to the case of dye-sensitized solar cells. TR for breakthrough technology fields requires the integration of a number of techniques to be able to handle the complexity of emergence and make it practicable to inform strategy and policy making. Such TR for technology fields can be further developed in a number of ways, by being 1) integrated with expert engagement to anticipate further multi-level evolutions -- e.g. in Carayannis et al. (2000), and 2) included in exploring multiple micro-level pathways such as open-ended roadmapping (Phaal et al. 2006; Phaal et al. 2012) to determine individual strategies to determine innovation pathways. A key challenge for TR is to evolve as part of on-going strategy or policy making. This requires "living TR." Our approach allows for regular updating and evaluation as part of such a "living 
TR” approach, and our future work will explore further this link to on-going future-oriented technology analyses.

\section{Acknowledgement}

We acknowledge support from the National High Technology Research and Development Program of China (Grant No. 2014AA015105), the Australian Research Council (ARC) under Discovery Project DP140101366, and the United States National Science Foundation (Award \#1527370 - Forecasting Innovation Pathways of Big Data \& analytics). Douglas K. R. Robinson (LISIS), participates in this research activity also as part of the Mapping Dynamics of Emerging Technologies project - MDET (ANR-10-ORA-007).

\section{References}

Allan, J., Carbonell, J. G., Doddington, G., Yamron, J., \& Yang, Y. (1998). Topic detection and tracking pilot study final report.

Blei, D. M. (2012). Probabilistic topic models. Communications of the ACM, 55(4), 77-84.

Bogard, D., \& Tiederman, W. (1986). Burst detection with single-point velocity measurements. Journal of Fluid Mechanics, 162, 389-413.

Carayannis, E. G., Alexander, J., \& Ioannidis, A. (2000). Leveraging knowledge, learning, and innovation in forming strategic government-university-industry (GUI) R\&D partnerships in the US, Germany, and France. Technovation, 20(9), 477-488.

Chen, C. (2006). CiteSpace II: Detecting and visualizing emerging trends and transient patterns in scientific literature. Journal of the American Society for Information Science and Technology, 57(3), 359-377.

Choi, C., \& Park, Y. (2009). Monitoring the organic structure of technology based on the patent development paths. Technological Forecasting and Social Change, 76(6), 754-768.

Etzkowitz, H., \& Leydesdorff, L. (1995). The Triple Helix--University-industry-government relations: A laboratory for knowledge based economic development. Easst Review, 14(1), 14-19.

Etzkowitz, H., \& Leydesdorff, L. (2000). The dynamics of innovation: from National Systems and "Mode 2" to a Triple Helix of university-industry-government relations. Research Policy, 29(2), 109-123.

Fenwick, D., Daim, T. U., \& Gerdsri, N. (2009). Value Driven Technology Road Mapping (VTRM) process integrating decision making and marketing tools: Case of Internet security technologies. Technological Forecasting and Social Change, 76(8), 1055-1077.

Garcia, M. L. (1997). Introduction to technology roadmapping: The semiconductor industry associations technology roadmapping process: Sandia National Labs., Albuquerque, NM (United States).

Garcia, M. L., \& Bray, O. H. (1997). Fundamentals of technology roadmapping: Sandia National Laboratories Albuquerque, NM.

Gerdsri, N., \& Kocaoglu, D. F. (2007). Applying the Analytic Hierarchy Process (AHP) to build a strategic framework for technology roadmapping. Mathematical and Computer Modelling, 46(7), 1071-1080.

Gerdsri, N., Vatananan, R. S., \& Dansamasatid, S. (2009). Dealing with the dynamics of technology roadmapping implementation: A case study. Technological Forecasting and Social Change, 76(1), 50-60.

Geum, Y., Lee, S., Kang, D., \& Park, Y. (2011). Technology roadmapping for technology-based product-service integration: A case study. Journal of Engineering and Technology Management, 28(3), 128-146.

Geum, Y., Lee, S., \& Park, Y. (2014). Combining technology roadmap and system dynamics simulation to support scenario-planning: A case of car-sharing service. Computers \& Industrial Engineering, 71, 37-49.

Geum, Y., Lee, H., Lee, Y., \& Park, Y. (2015). Development of data-driven technology roadmap considering dependency: An ARM-based technology roadmapping. Technological Forecasting and Social Change, 91, 264-279.

Guo, Y., Ma, T., Porter, A. L., \& Huang, L. (2012). Text mining of information resources to inform Forecasting Innovation Pathways. Technology Analysis \& Strategic Management, 24(8), 843-861.

Hamilton, J. D. (1994). Time series analysis (Vol. 2): Princeton university press Princeton.

Huang, L., Guo, Y., Porter, A. L., Youtie, J., \& Robinson, D. K. (2012). Visualising potential innovation pathways in a workshop setting: the case of nano-enabled biosensors. Technology Analysis \& Strategic Management, 24(5), 527-542. 
Huang, L., Zhang, Y., Guo, Y., Zhu, D., \& Porter, A. L. (2014). Four dimensional Science and Technology planning: A new approach based on bibliometrics and technology roadmapping. Technological Forecasting and Social Change, 81, 39-48.

Jeong, Y., \& Yoon, B. (2015). Development of patent roadmap based on technology roadmap by analyzing patterns of patent development. Technovation, 39, 37-52.

Kim, Y., Tian, Y., Jeong, Y., Jihee, R., \& Myaeng, S.-H. (2009). Automatic discovery of technology trends from patent text. Paper presented at the Proceedings of the 2009 ACM symposium on Applied Computing.

Kostoff, R. N., \& Schaller, R. R. (2001). Science and technology roadmaps. Engineering Management, IEEE Transactions on, 48(2), 132-143.

Kostoff, R. N., Toothman, D. R., Eberhart, H. J., \& Humenik, J. A. (2001). Text mining using database tomography and bibliometrics: A review. Technological Forecasting and Social Change, 68(3), 223-253.

Kostoff, R. N., Boylan, R., \& Simons, G. R. (2004). Disruptive technology roadmaps. Technological Forecasting and Social Change, 71(1), 141-159.

Kostoff, R. N., Briggs, M. B., Solka, J. L., \& Rushenberg, R. L. (2008). Literature-related discovery (LRD): Methodology. Technological Forecasting and Social Change, 75(2), 186-202.

Kostoff, R. N. (2014). Literature-related discovery: common factors for Parkinson's Disease and Crohn's Disease. Scientometrics, 100(3), 623-657.

Kostoff, R. N., \& Patel, U. (2015). Literature-related discovery and innovation: Chronic kidney disease. Technological Forecasting and Social Change, 91, 341-351.

Lee, S., \& Park, Y. (2005). Customization of technology roadmaps according to roadmapping purposes: Overall process and detailed modules. Technological Forecasting and Social Change, 72(5), 567-583.

Lee, S., Yoon, B., Lee, C., \& Park, J. (2009a). Business planning based on technological capabilities: Patent analysis for technology-driven roadmapping. Technological Forecasting and Social Change, 76(6), 769-786.

Lee, S., Yoon, B., \& Park, Y. (2009b). An approach to discovering new technology opportunities: Keyword-based patent map approach. Technovation, 29(6), 481-497.

Liben-Nowell, D., \& Kleinberg, J. (2007). The link - prediction problem for social networks. Journal of the American Society for Information Science and Technology, 58(7), 1019-1031.

Park, H. W., Hong, H. D., \& Leydesdorff, L. (2005). A comparison of the knowledge-based innovation systems in the economies of South Korea and the Netherlands using Triple Helix indicators. Scientometrics, 65(1), 3-27.

Phaal, R., Farrukh, C. J., \& Probert, D. R. (2004). Technology roadmapping-a planning framework for evolution and revolution. Technological Forecasting and Social Change, 71(1), 5-26.

Phaal, R., Farrukh, C. J., \& Probert, D. R. (2006). Technology management tools: concept, development and application. Technovation, 26(3), 336-344.

Phaal, R., Kerr, C., Oughton, D., \& Probert, D. (2012). Towards a modular toolkit for strategic technology management. International Journal of Technology Intelligence and Planning, 8(2), 161-181.

Porter, A. L., \& Cunningham, S. W. (2004). Tech mining: exploiting new technologies for competitive advantage (Vol. 29): John Wiley \& Sons.

Porter, A. L., \& Cunningham, S. W. (2005). Tech mining. Competitive Intelligence Magazine, 8(1), 30-36.

Porter, A. L., Guo, Y., Huang, L., \& Robinson, D. K. (2010). Forecasting innovation pathways: The case of nano-enhanced solar cells. Paper presented at the International conference on technological innovation and competitive technical intelligence.

Porter, A. L., Cunningham, S. W., \& Sanz, A. (2013). Extending the FIP (Forecasting Innovation Pathways) approach through an automotive case analysis. Paper presented at the Technology Management in the IT-Driven Services (PICMET), 2013 Proceedings of PICMET'13:.

Probert, D., \& Radnor, M. (2003). Technology roadmapping: frontier experiences from industry-academia consortia. Research Technology Management, 46(2), 27.

Rafols, I., Porter, A. L., \& Leydesdorff, L. (2010). Science overlay maps: A new tool for research policy and library management. Journal of the American Society for Information Science and Technology, 61(9), 1871-1887.

Rantanen, K., \& Domb, E. (2010). Simplified TRIZ: new problem solving applications for engineers and manufacturing professionals: CRC press. 
Resnick, P., Iacovou, N., Suchak, M., Bergstrom, P., \& Riedl, J. (1994). GroupLens: an open architecture for collaborative filtering of netnews. Paper presented at the Proceedings of the 1994 ACM conference on Computer supported cooperative work.

Resnick, P., \& Varian, H. R. (1997). Recommender systems. Communications of the ACM, 40(3), 56-58.

Rip, A., \& Kemp, R. (1998). Technological change: Battelle Press.

Robinson, D., Zhou, X., Guo, Y., \& Porter, A. (2013a). Nano-enabled Drug Delivery-Capturing the state of the art and forecasting innovation pathways. Paper presented at the Portland International Conference on Management of Engineering and Technology (PICMET), San Jose, CA.

Robinson, D. K., Huang, L., Guo, Y., \& Porter, A. L. (2013b). Forecasting Innovation Pathways (FIP) for new and emerging science and technologies. Technological Forecasting and Social Change, 80(2), 267-285.

Tierney, R., Hermina, W., \& Walsh, S. (2013). The pharmaceutical technology landscape: A new form of technology roadmapping. Technological Forecasting and Social Change, 80(2), 194-211.

Tran, T. A., \& Daim, T. (2008). A taxonomic review of methods and tools applied in technology assessment. Technological Forecasting and Social Change, 75(9), 1396-1405.

Walsh, S. T. (2004). Roadmapping a disruptive technology: A case study: The emerging microsystems and top-down nanosystems industry. Technological Forecasting and Social Change, 71(1), 161-185.

Walsh, S. T., Boylan, R. L., McDermott, C., \& Paulson, A. (2005). The semiconductor silicon industry roadmap: epochs driven by the dynamics between disruptive technologies and core competencies. Technological Forecasting and Social Change, 72(2), 213-236.

Waltman, L., van Eck, N. J., \& Noyons, E. C. (2010). A unified approach to mapping and clustering of bibliometric networks. Journal of Informetrics, 4(4), 629-635.

Winebrake, J. J. (2004). Alternate energy: Assessment and implementation reference book: The Fairmont Press, Inc.

Yoon, B., \& Park, Y. (2005). A systematic approach for identifying technology opportunities: Keyword-based morphology analysis. Technological Forecasting and Social Change, 72(2), 145-160.

Zadeh, L. A. (1965). Fuzzy sets. Information and control, 8(3), 338-353.

Zhang, Y., Guo, Y., Wang, X., Zhu, D., \& Porter, A. L. (2013). A hybrid visualisation model for technology roadmapping: bibliometrics, qualitative methodology and empirical study. Technology Analysis \& Strategic Management, 25(6), 707-724.

Zhang, Y., Porter, A. L., Hu, Z., Guo, Y., \& Newman, N. C. (2014a). “Term clumping” for technical intelligence: A case study on dye-sensitized solar cells. Technological Forecasting and Social Change, 85, 26-39.

Zhang, Y., Zhou, X., Porter, A. L., \& Gomila, J. M. V. (2014b). How to combine term clumping and technology roadmapping for newly emerging science \& technology competitive intelligence:"problem \& solution” pattern based semantic TRIZ tool and case study. Scientometrics, 101(2), 1375-1389.

Zhang, Y., Zhou, X., Porter, A. L., Gomila, J. M. V., \& Yan, A. (2014c). Triple Helix innovation in China's dye-sensitized solar cell industry: hybrid methods with semantic TRIZ and technology roadmapping. Scientometrics, 99(1), 55-75.

Zhang, Y., Chen, H., Zhang, G., Porter, A. L., Zhu, D., \& Lu, J. (2015a). Topic Analysis and Forecasting for Science, Technology and Innovation: Methodology and a Case Study. Technological Forecasting and Social Change, to appear.

Zhang, Y., Chen, H., Zhang, G., Zhu, D., \& Lu, J. (2015b). Multiple Science Data-Oriented Technology Roadmapping Method. Paper presented at the Portland International Center for Management of Engineering and Technology, Portland, USA.

Zhou, X., Zhang, Y., Porter, A. L., Guo, Y., \& Zhu, D. (2014). A patent analysis method to trace technology evolutionary pathways. Scientometrics, 100(3), 705-721.

Zhu, D., \& Porter, A. L. (2002). Automated extraction and visualization of information for technological intelligence and forecasting. Technological Forecasting and Social Change, 69(5), 495-506. 
Yi Zhang is a dual-degree Ph.D. candidate at the Decision Systems and e-Service Intelligence Research Laboratory, Centre for Quantum Computation and Intelligent System, Faculty of Engineering and Information Technology, University of Technology Sydney, Australia, and the School of Management and Economics, Beijing Institute of Technology, China. His specialty is text mining and technology innovation management, especially technological assessment and forecasting with the combination of qualitative and quantitative methodologies.

Douglas K. R. Robinson is an expert in innovation analysis. Originally trains as a physicist and space scientist, Douglas has a $\mathrm{PhD}$ in innovation studies and technology assessment and experience in a number of Innovation management institutions in Europe as well as in the private sector. His research and consultancy activities follow 3 interlinked axes: (1) Assessment and foresight of industrial change and business model innovation relating to breakthrough technologies (such as additive manufacturing, nanomaterials in the food sector and bio-printing), (2) Policy and strategy intelligence on research and innovation, (3) Projecting innovation and impact pathways through the analysis of value chains, regulatory factors, finance and potential societal/market uptake.

Alan L Porter is Professor Emeritus of Industrial \& Systems Engineering, and of Public Policy, at Georgia Institute of Technology, where he remains Co-director of the Technology Policy and Assessment Center. He is also Director of R\&D for Search Technology, Inc., Norcross, GA. He is author of some 220 articles and books. Current research emphasizes measuring, mapping, and forecasting ST\&I knowledge diffusion patterns.

Donghua Zhu is currently a Professor and the Associate Dean of the School of Management and Economics, and the Director of the Knowledge Management and Data Analysis Laboratory, at Beijing Institute of Technology, China. His main academic research fields include science and technology data mining, technology innovation management, technology forecasting and management. His current research emphasizes big data analytics.

Guangquan Zhang received his Ph.D. degree in applied mathematics from Curtin University of Technology, Perth, Australia, in 2001. He is currently an Associate Professor in the Faculty of Engineering and Information Technology, and the Co-director of the Decision Systems and e-Service Intelligence Research Laboratory, Centre for Quantum Computation and Intelligent Systems, at University of Technology Sydney. His main research interests include multi-objective and group decision making, decision support system tools, fuzzy measure and optimization, and uncertain information processing.

Jie Lu received the Ph.D. from Curtin University of Technology, Perth, Australia, in 2000. She is currently a Professor and the Associate Dean Research (Acting) in the Faculty of Engineering and Information Technology, and the Director of the Decision Systems and e-Service Intelligence Research Laboratory, Centre for Quantum Computation and Intelligent Systems, at University of Technology Sydney, Australia. Her main research interests include decision making modeling, decision support system tools, uncertain information processing, recommender systems, and e-Government and e-Service intelligence. 